\title{
L'uso del gerundio con \\ i verbi di percezione \\ gardenesi
}

Jan Casalicchio

\section{Introduzione}

Questo lavoro è basato sulla tesi di laurea da me discussa nel luglio 2009 presso la Facoltà di Lettere e Filosofia dell'Università di Trento, in cui ho esaminato i diversi tipi di strutture che si possono utilizzare in gardenese con $i$ verbi di percezione come vedere o sentire. ${ }^{1}$ In questo articolo prenderò in esame solo una delle costruzioni che possono dipendere da questi verbi, ossia le frasi in cui il verbo subordinato è espresso - invece che all'infinito come in italiano - al gerundio. Nell'ambito del mondo romanzo l'uso di una struttura di questo tipo con i verbi di percezione non è una caratteristica esclusiva del ladino, perché esso risale probabilmente al latino volgare e oggi è comune - seppur con dei tratti diversi - a lingue come lo spagnolo, il francese, il sardo e il romeno, oltre alle varietà ladine delle valli di Badia (ivi compresa Marebbe) e Gardena. La costruzione è invece assente dall'italiano contemporaneo, ma non era infrequente nei primi secoli della letteratura italiana. ${ }^{2}$

1 Casalicchio, Jan: La convivenza di elementi arcaici e innovaz̧oni nella costružione percettiva gardenese, Università degli Studi di Trento 2009; vorrei qui ringraziare il relatore, prof. Giampaolo SALvI, e la correlatrice, prof.ssa Patrizia Cordin, per il loro aiuto e appoggio. La redazione di quest'articolo ha beneficiato di importanti suggerimenti da parte delle prof.sse Patrizia Cordin e Heidi SiLLER-RungGaLdier. La tesi è dedicata a mia nonna.

2 Alcuni autori pensano che in italiano antico la gerundiva con i verbi di percezione fosse un francesismo (cf. per esempio EgerLand 2010, 920), ma sembra più probabile uno sviluppo autoctono, cf. ŠKERLJ 1926 (in particolare alle pagine 215-219). 
La gerundiva costituisce però solo una delle possibili costruzioni che possono dipendere da un verbo di percezione. Complessivamente, infatti, in gardenese si contano ben otto strutture sintattiche utilizzabili con questi verbi, e il quadro generale si presenta ancor'oggi in mutamento e ricco di innovazioni peculiari al ladino, in parte per uno sviluppo interno, basato sul materiale proprio di questa varietà, in parte invece per probabile influsso delle due lingue tetto del ladino dolomitico, l'italiano e il tedesco. La concomitanza di questi diversi fattori ha fatto sì che oggi l'insieme delle costruzioni percettive gardenesi rappresenti un unicum all'interno del mondo romanzo. ${ }^{3}$

La descrizione della costruzione percettiva gardenese si basa in parte sull'analisi di un corpus di testi consultabili in rete grazie al progetto TALES, ${ }^{4} \mathrm{e}$ in parte sulle risposte fornite da quindici parlanti nativi gardenesi a un questionario approntato appositamente per questo argomento. Alcune frasi, inoltre, sono tratte dalle carte dell' $A$ ID-II, che mi sono state gentilmente messe a disposizione dal professor Hans Goebl. In questo articolo mi concentrerò sulle proposizioni gerundive, sia perché costituiscono la struttura che ricorre più frequentemente con i verbi di percezione, sia perché ci permettono un confronto con altre lingue del mondo romanzo, per cogliere così gli aspetti comuni e le differenze che intercorrono in un ambito particolare della grammatica.

\section{La costruzione percettiva romanza}

Nel latino classico i verbi di percezione potevano introdurre una proposizione con il participio (video puerum currentem) o all'infinito (video puerum currere), che si differenziavano semanticamente: l'uso del participio indicava una percezione diretta da parte del soggetto, mentre l'infinitiva sottolineava l'aspetto cognitivo e poteva descrivere anche una percezione indiretta, di cui si viene a conoscenza per altri motivi. Questo sistema subì però delle notevoli modifiche già in epoca tardoantica: l'infinitiva semplice fu sostituita da una completiva introdotta da quod /

3 Sebbene gran parte delle caratteristiche che descriverò d'ora in avanti siano comuni anche al badiotto e al marebbano, in questo articolo mi limiterò esclusivamente alla situazione del gardenese; potremo fare solo pochi riferimenti alle altre varietà ladine, perché mancano studi dedicati ai verbi di percezione di questi sistemi linguistici.

4 TALES $=$ "Trattamento Automatico delle Lingue Ladina e Sarda". Consultabile al sito <http://corpuslad.ladintal.it/applications/textanalysis/sitecorpuslad/index.jsp?_VP_V_ID=2838195> (data dell'ultima consultazione: 7.11 .2011$)$. 
quia (nelle lingue romanze che o que), mentre l'uso del participio sopravvisse fino al medioevo, subendo però sempre di più la concorrenza del gerundio ${ }^{5} \mathrm{o}$ di una proposizione infinitiva. ${ }^{6}$ Risale invece probabilmente al medioevo lo sviluppo della cosiddetta pseudorelativa (Vedo Andrea che mangia $i$ biscotti).

Se si confrontano le costruzioni percettive oggi esistenti nelle varie lingue romanze, si può vedere come alcuni sviluppi medioevali siano stati mantenuti in tutta la Romània, mentre altre strutture sono sopravvissute in un gruppo più ristretto di lingue. In particolare, le lingue che hanno conservato la costruzione percettiva al gerundio sono lo spagnolo, il francese, il romeno, il sardo e alcune varietà del romancio e del ladino dolomitico: in queste lingue la gerundiva alterna solitamente con l'infinitiva.

In spagnolo, la costruzione usata con maggior frequenza è l'infinitiva, ma è possibile ricorrere anche a una proposizione al gerundio (Oi a Juan tocando la guitarra, "Sentii Juan suonare la chitarra"). Le diverse costruzioni divergono però sia nella sintassi, sia nella semantica: secondo JÄGER (1993, 304-305), l'infinitiva va interpretata come un blocco unico imperniato sul verbo all'infinito. La proposizione gerundiva e la pseudorelativa, invece, ricoprirebbero la funzione di complemento predicativo e descriverebbero il complemento oggetto del verbo reggente ${ }^{7}-$ secondo la linguista tedesca - in uno stato contemporaneo, o coinvolto in un evento contemporaneo rispetto al tempo del verbo di percezione. Per questo motivo la gerundiva non può essere usata quando l'oggetto non è espresso (*Oi tocando la guitarra, "Sentii suonare la chitarra"). Quest'ultima costruzione è inoltre legata a un'interpretazione aspettuale, perché il gerundio rappresenta per sua natura un'azione in svolgimento, che non implica né l'inizio né il termine dell'azione stessa, e perciò è incompatibile con verbi di stato o puntuali, diversamente da quanto accade con l'infinitiva.

5 Per maggiori informazioni sugli sviluppi tardoantichi e medioevali delle strutture participiali e gerundive (anche con i verbi di percezione), cf. ŠKERLJ 1926 e TeKAVČIĆ 1972, 250-253 e 383-387.

6 È importante notare come l'infinitiva romanza diverga sia strutturalmente, sia semanticamente dall'infinitiva del latino classico (cf. SALVI, ms. inedito).

7 Quando il verbo di percezione è in forma attiva, il suo complemento oggetto costituisce anche il soggetto semantico (ma non sintattico) del gerundio o infinito (diversamente dalle frasi al passivo, dove è il soggetto del verbo principale a coincidere con il soggetto semantico del gerundio/infinito). Nel corso di quest'articolo utilizzerò alternativamente le due etichette anche se si riferiscono allo stesso elemento, a seconda delle esigenze esplicative. 
Il francese presenta una situazione simile allo spagnolo. Va però notato come il gerundio spagnolo corrisponda al cosiddetto participe présent del francese. ${ }^{8}$ In questo modo si possono evitare quelle ambiguità che esistono in alcune frasi spagnole: mentre una frase come He visto a Sheila salendo de l'universidad è ambigua, perché il gerundio può essere riferito sia al soggetto del verbo principale, sia al soggetto semantico del gerundio ("Ho visto Sheila uscendo dall'università" vs. "Ho visto Sheila uscire dall'università"), in francese avremo due frasi diverse: J'ai vu Sheila en sortant de l'université e J'ai vu Sheila sortant de l'université.

Rispetto a queste due lingue, il romeno presenta una situazione assai diversa: dal momento che in questa lingua l'infinito è quasi inesistente e limitato a pochissime funzioni, la gerundiva costituisce la proposizione utilizzata con maggior frequenza per indicare una percezione diretta. Anche in romeno la proposizione al gerundio può però risultare ambigua. Così una frase come Pe respectabilia madam Trahanache am văzut-o ieşind de la Catavencu può essere letta in due modi: "La rispettabile signora Trahanache ho visto uscire (oppure: mentre uscivo) da Cațavencu". ${ }^{10}$

Prima di analizzare la situazione del ladino gardenese, vediamo quale sia la situazione delle due lingue vicine, italiano e tedesco, non solo sincronicamente, ma anche nella dimensione diacronica, per vedere se il loro modello abbia in qualche modo influenzato la struttura percettiva del gardenese. Nel medioevo, in italiano era possibile ricorrere al gerundio o all'infinito con le stesse modalità dello spagnolo contemporaneo. In seguito, però, si affermò sempre di più la propensione a ricorrere esclusivamente all'infinito, parallelamente alla tendenza a impiegare il gerundio solo nei casi di coreferenza con il soggetto della frase principale. Le altre varietà ladine (o retoromanze) mostrano un quadro diversificato: in romancio in alcuni casi è possibile utilizzare la gerundiva, benché la scelta non marcata sia rappresentata anche qui dall'infinito. Il friulano e le varietà ladino-dolomitiche meridionali (fassano e livinallese) hanno invece generalizzato l'uso dell'infinito, forse su influsso dell'italiano, e come quest'ultimo non ammettono il gerundio in questa funzione.

${ }^{8}$ La grammatica francese distingue tra il participe (per es. sortant) e il gérondif (en sortant): diacronicamente entrambe le forme derivano dal gerundio latino, e solo a partire dall'epoca moderna le due forme vengono differenziate in modo stabile mediante l'aggiunta della preposizione en al gerundio; cf. LAMBERTZ 1987, 175-186, in cui si trova anche una descrizione della gerundiva francese dopo i verbi di percezione.

9 Per il francese, cf. LAMBERTz 1987.

10 Sul rumeno cf. Siller-Runggaldier 1997, 316-317, da cui proviene anche l'esempio che la studiosa cita da Edelstein 1972, n. 18, 21. 
Nelle lingue germaniche, invece, non esiste il gerundio, ma esiste il participio; già in antico alto tedesco la struttura percettiva era formata principalmente per mezzo dell'infinito, anche se non sono esclusi alcuni ricorsi al participio, come avviene ancor oggi in inglese (I hear him laughing). In tedesco si ricorre solitamente all'infinito, ma è possibile trovare delle frasi formate con il Partizip Präsens anche nel tedesco contemporaneo (per esempio Ich sah ihn schlafend, "Lo vidi dormiente"), sebbene risultino di stile antiquato. ${ }^{11}$

\section{La costruzione percettiva gardenese e badiotta}

Rispetto al panorama romanzo, le varietà settentrionali del ladino dolomitico presentano una situazione del tutto particolare. Mentre infatti fassano e livinallese si comportano come i vicini dialetti trentini e bellunesi (uso esclusivo dell'infinito semplice o introdotto dalla preposizione $a$ ), in gardenese e badiotto si è avuta un'evoluzione opposta, per cui è possibile ricorrere al gerundio e non all'infinito, una situazione che assomiglia al quadro del romeno. ${ }^{12}$ Le cause di questo sviluppo però sono differenti: se in romeno l'impossibilità di utilizzare un'infinitiva con i verbi di percezione è legata a un fattore strutturale che si pone in un contesto di progressiva marginalizzazione dell'infinito, in ladino la situazione è più complessa, poiché l'infinito è di per sé assai vitale. Dal momento che i primi documenti ladini di una certa lunghezza risalgono al XIX secolo, ${ }^{13}$ non ci è possibile delineare il percorso che ha portato alla peculiare situazione odierna. In ogni caso, si possono individuare due possibili linee di sviluppo:

- il ladino non ha mai usato l'infinito in questa funzione;

- in epoca medievale l'infinito veniva usato anche in ladino accanto al gerundio, ma poi è stato completamente sostituito da quest'ultimo.

Tenendo conto della vitalità di cui gode oggi complessivamente l'infinito in ladino, penso che si possa propendere per la prima ipotesi. Infatti, se un tempo il gardenese e il badiotto avessero permesso anche l'uso del gerundio, in maniera simile

${ }^{11}$ Da una sommaria verifica su Internet risulta come ancor oggi si possano formare delle frasi con il participio dipendente da un verbo di percezione (per esempio in alcuni blog), per quanto si tratti di un uso molto limitato.

12 Per le varietà ladine in generale cf. le carte dell'ALD-II, e per il badiotto in particolare GASSER 2000.

13 I primi testi certamente ladini sono due proclami del principe vescovo di Bressanone del XVII secolo, rispettivamente in fassano e fodom (cf. PlangG 1985). Non è ancora stato stabilito in modo definitivo, invece, se alcuni versi di Oswald von Wolkenstein (1445) siano stati formulati in gardenese, cf. KuEN 1979. 
a quanto accade oggi in spagnolo, non si vede come una forma meno usata, e oltretutto più marcata, avrebbe potuto eliminare e sostituire l'infinito anche in quei contesti che sono inaccessibili al gerundio spagnolo (per cui cf. infra). Come nel caso dell'ordine delle parole di tipo V2 di gardenese e badiotto, ${ }^{14}$ anche per questo fenomeno è possibile che vi sia stato un qualche influsso dei limitrofi dialetti tedeschi (perlomeno nella conservazione di questo tratto), e che forse i parlanti abbiano posto in relazione le strutture percettive introdotte dalla congiunzione wie o dalla preposizione $b e i+$ infinito sostantivato con la loro costruzione gerundiva: ${ }^{15}$

(1) Ich sehe, wie die Frau das Hemd bügelt.

"Vedo, come la signora stira la camicia."

(2) Ich sehe den Direktor beim Zeitung Lesen.

"Vedo il direttore leggere il giornale."

D'altra parte, una struttura simile all'infinito sostantivato si ritrova in molti dialetti dell'Italia settentrionale, in cui un verbo di percezione può essere seguito dalla costruzione $a+$ infinito; ${ }^{16}$ non si può dunque escludere che la gerundiva ladina abbia subito qualche influenza da queste strutture che hanno una semantica simile. Ci troviamo comunque nel campo delle ipotesi, e la scarsità di dati sui periodi precedenti il XIX secolo rende al momento impossibile una spiegazione convincente dell'origine di questa peculiarità.

14 Cf. BENINCÀ 1988, la quale si è occupata della regola del verbo in seconda posizione nel ladino delle valli di Gardena e Badia. La linguista padovana ha rimarcato che questo fenomeno non può essere considerato semplicemente come un calco sintattico dell'ordine V2 del tedesco, e che una regola simile a quella del ladino odierno vigeva anche in italiano antico. La struttura a V2 del gardenese e badiotto è quindi la conservazione di un tratto proprio di queste due varietà, conservazione dovuta - o almeno favorita - all'analogia con la situazione tedesca.

${ }^{15}$ Il gardenese fa però già uso di una struttura parallela a quella dell'esempio (1), in cui al wie corrisponde coche (entrambi con il significato "come”), cf. (7).

${ }^{16}$ La costruzione $a+$ infinito, che rispetto alla costruzione tedesca beim + infinito mantiene maggiormente la sua natura verbale, presenta in molti dialetti delle limitazioni sintattiche simili a quelle del gerundio spagnolo, e in alcune varietà, tra cui il fodom, ha soppiantato quasi del tutto l'infinito semplice; si vedano questi esempi (convenzionalmente, i punti di domanda in apice prima di una parentesi indicano che l'elemento seguente è quasi obbligatorio; l'asterisco invece qui segnala che l'elemento tra parentesi è del tutto agrammaticale):

(i) L veighe très ?? (a) mangé douciarie. (Fodom: "Lo vedo sempre mangiare dolciumi.")

(ii) Luca al sent $(* a)$ sunà $u$ cant da la banda. (Villa d'Ogna, BG: "Luca sente suonare un canto dalla banda.") In (i) la struttura con l'infinito semplice è del tutto marginale, perché con i verbi di percezione solitamente si ricorre all'infinito introdotto dalla preposizione $a$; in (ii), invece, si vede che quando l'infinito è seguito da un complemento d'agente è necessario usare, almeno nella varietà di Villa d'Ogna, l'infinito semplice. Cf. Casalicchio/Modena (ms. inedito), da cui sono tratti i due esempi. 
La proposizione gerundiva non costituisce comunque l'unica costruzione possibile con i verbi di percezione. In gardenese, infatti, se ne contano ben otto tipi diversi, che si possono suddividere in base all'assenza o presenza di un verbo subordinato. Al primo gruppo appartengono i complementi predicativi composti da un sintagma aggettivale (es. 3), un sintagma preposizionale (4) e una struttura al participio passato (5). Le costruzioni con verbo subordinato si possono invece suddividere tra strutture che ospitano un verbo finito (completive introdotte dai complementatori che o coche, e frasi relative), esemplificate rispettivamente negli esempi (6-8), e strutture con un verbo in forma non personale (9-10). Fanno parte di questo gruppo le gerundive e le infinitive; queste ultime sono rare in gardenese (e non previste dalla grammatica normativa), ma non del tutto assenti:

(3) N la udova bën for da sëula $i$ ultims ani, no pa? (Lëtr. $)^{17}$

"La si vedeva quasi sempre da sola negli ultimi anni, o no?"

(4) Te chëla sëntel na man ciauda y mujela sun si ciavëi (Lib. rel. 3)

"In quel momento sente una mano calda e morbida sui suoi capelli."

(5) Na fancela l'à udù sentà pra fuech, y ti cialàn fit, dìjela [...] (Bib. sand. C O)

"Un'ancella l'ha visto seduto presso il fuoco, e guardandolo fisso, dice [...]"

(6) I fova dalonc, ma mpo udovi che Jan sla rijova. (Laur. deb. 3)

"Erano lontani, ma comunque vedevano che Jan se la rideva."

(7) Ora dla majon numer 2 àuden coche Einstein sona la vidula. (Fis.)

"Fuori dalla stanza numero 2 si sente come [= che] Einstein suona il violino."

(8) Totl de 'n paur, $[\ldots]$ n'ès-a mè̀ udù na persona che raita? (N puecb)

"Stupido di un contadino, $[\ldots]$ non hai mai visto una persona che cavalca?"

(9) L ciof ova udú n di passán na carovana. (L pitl pr.)

"Il fiore aveva visto un giorno passare una carovana."

(10) Y la stëila che i ova udù levé, jiva a d'ëi dan ora [...]. (Bib. sand. C O)

"E la stella che avevano visto alzarsi, andava davanti a loro [...]."

${ }^{17}$ Le citazioni dal CorpusLad sono riprese fedelmente, mantenendo eventuali errori di digitazione e l'ortografia originaria per i testi più antichi. 


\section{La costruzione con il gerundio}

\subsection{I verbi di percezione}

Nella varietà ladina della Val Gardena, una proposizione gerundiva può essere introdotta solo dai tre verbi di percezione usati con maggior frequenza: audi, sentì e udëi ("udire", "sentire [con il tatto]", "vedere"). Esistono anche altri verbi di percezione, più specifici e meno frequenti, che però devono essere combinati con altre costruzioni, in primis le completive con coche o che (cf. supra, \3). Si tratta per esempio di cialé e della sua variante sintagmatica cialé pro ("guardare"), cuché ("guardare non osservati, spiare") e scuté sù ("ascoltare").

La possibilità di ricorrere a una gerundiva è dunque limitata ai tre verbi usati più frequentemente e più generici dal punto di vista semantico. ${ }^{18}$ L'uso di questi tre verbi non ha limitazioni; il verbo principale può essere espresso in qualunque tempo o modo verbale, compreso un gerundio (con una vicinanza quindi di due gerundi):

(11) I ujins s'la cuinova udan Noé zumpran cb'la ercia coche na gran arca. (Bib. pit. VT)

"I vicini se la ridevano vedendo Noè lavorare quel granaio come [= sotto forma di] una grande arca."

Di per sé, una proposizione gerundiva è compatibile anche con un verbo di percezione al passivo:

(12) L lere ie unì udù mucian cun l motor. (Q21 a)

"Il ladro è stato visto fuggire con la moto."

L'esempio (12) però non viene accettato unanimemente dai parlanti; infatti in gardenese esista la tendenza a evitare $i$ verbi al passivo, per cui si preferisce ricorrere a una struttura impersonale con il pronome $n$ (in proclisi) / -(e) $n$ (in enclisi). Questo vale anche per i verbi di percezione:

(13) Nfati, $n$ valgun dis do vëijen Tone lauran pra bera Franæl. (Bels. 1)

"Infatti, alcuni giorni dopo si vede Tone lavorare presso bera Franzl."

\footnotetext{
${ }^{18}$ Va notato come in tedesco gli unici verbi che permettono un complemento all'infinito siano bören, sehen e füblen/spüren, che corrispondono ai tre verbi del gardenese. Non si può comunque escludere uno sviluppo parallelo e indipendente, che corrisponda dunque in qualche modo a una tendenza universale a limitare costruzioni frasali in funzione di complemento predicativo solo ai verbi più generici. L'italiano, invece, come risulta evidente da SKYTTE (1983, 251-252), è molto meno restrittivo su questo punto.
} 
In generale, comunque, i parlanti hanno mostrato comportamenti discordi nella valutazione di frasi con il verbo di percezione al passivo: se alcuni le rifiutavano completamente, altri invece le accettavano con più facilità, soprattutto quando venivano invitati a immaginare tali frasi nel contesto di un articolo di giornale o di un servizio televisivo. Evidentemente l'uso di forme verbali al passivo è più diffuso proprio nel linguaggio giornalistico, non solo in ladino ma anche in italiano o tedesco.

\subsection{II soggetto semantico del gerundio}

Passiamo ora ad analizzare l'elemento nominale o pronominale che funge da complemento oggetto del verbo di percezione e contemporaneamente costituisce semanticamente il soggetto dell'evento descritto dalla frase al gerundio. In quest'ambito non sembrano esistere limitazioni; se gli elementi più frequenti sono i sintagmi nominali ( $\mathrm{SN}$ ) con testa nominale (definiti tradizionalmente “sostantivi”) e i pronomi clitici, sporadicamente appare anche una serie di altri tipi pronominali, come i dimostrativi, i pronomi personali tonici, i riflessivi, gli indefiniti e i pronomi relativi:

(14) Chësta (sc. la man) sentivel striscian bel plan ju per l cë y jan a se fermé sun si sciabla ciancia. (Lib. rel. 3)

"Questa (sc. la mano) sentiva scivolare pian piano giù dalla testa e andare a fermarsi sulla sua spalla sinistra."

Uno dei tratti più caratteristici e innovativi del gardenese riguarda la possibilità di formulare una costruzione gerundiva anche senza un soggetto semantico. $\mathrm{Si}$ assiste così a casi in cui il gerundio si trova a dipendere direttamente da un verbo di percezione. Si tratta di una peculiarità unica del gardenese, e del tutto inaspettata per chi conosca il quadro delle altre varietà romanze. In gardenese la gerundiva priva di referente non è un fenomeno eccezionale, perché è ben attestata sia nel corpus da noi analizzato, sia nei questionari sottoposti agli informatori. La maggior parte di questi esempi contiene il verbo audi; ciò probabilmente non è dovuto a una vera e propria regola, ma è spiegabile con la maggiore indeterminatezza della percezione uditiva: infatti è molto più difficile individuare il responsabile di qualcosa che si percepisce con l'udito, che non il partecipante a un evento che si vede. 
Si confrontino gli esempi seguenti:

(15a) Ntan i prims ani de vita ova Gina donca mé audí rujenan tudësch. (Lëtr.)

"Durante i primi anni di vita Gina quindi aveva sentito solo parlare tedesco."

(16a) Ie vë̈je ciacian l cërf. (Q20a)

"Vedo cacciare il cervo."

In entrambi gli esempi manca il soggetto semantico del gerundio; in (15a) si potrebbe inserire un elemento come duc ("tutti"), duta la persones ("tutte le persone") mentre in (16a) si potrebbe aggiungere zachei ("qualcuno"), n iagher / ciaciadëur ("un cacciatore"), n lëuf (“un lupo"), etc.:

(15b) Ntan i prims ani de vita ova Gina donca audí duc rujenan tudësch.

"Durante i primi anni di vita Gina aveva quindi sentito tutti parlare tedesco."

(16b) Ie vë̈je n iagher / zachei ciacian l cërf.

"Vedo un cacciatore / qualcuno cacciare il cervo."

La mancanza del soggetto semantico dunque non è dovuta a fatti sintattici, bensì alla scelta di porre in primo piano l'evento descritto nella costruzione percettiva: in (15a) il fatto che Gina nei primi anni di vita conobbe solo il tedesco, e in (16a) il fatto che il cervo viene cacciato. Se il soggetto semantico venisse invece espresso, l'accento cadrebbe inevitabilmente su di esso, sviando così l'attenzione dall'evento in sé. ${ }^{19}$

È degno di nota il fatto che la costruzione gerundiva è grammaticale anche con i verbi cosiddetti zerovalenti, come per esempio i verbi meteorologici, che sono sempre privi di un soggetto semantico, anche nelle lingue - come

${ }^{19}$ In apparenza, questa caratteristica potrebbe sembrare collegabile alla possibilità di non indicare il soggetto pronominale con i verbi flessi in italiano e in parte in gardenese (Vado anziché Io vado). Sono convinto però che questi due fenomeni vadano tenuti distinti, perché con i verbi flessi il soggetto è comunque recuperabile dalla morfologia flessiva o dal contesto (nei casi di terza persona). Con i verbi di percezione, invece, in assenza del soggetto semantico solitamente il contesto non permette di risalire alla sua identità, e infatti frasi di questo tipo solitamente possono essere parafrasate da frasi completive che hanno come soggetto un quantificatore come tutti, qualcuno (o nessuno se la frase è negata); per es. Sento cantare in giardino = Sento che qualcuno canta in giardino.

Inoltre, il confronto con il francese dimostra come i due fenomeni non siano collegati: in francese, infatti, i verbi flessi devono avere obbligatoriamente un soggetto espresso, mentre nelle costruzioni percettive all'infinito questo può mancare: J'entends chanter (“Sento cantare"). 
il gardenese - in cui le forme flesse di questi verbi sono accompagnate da un pronome espletivo (l pluef, come in tedesco es regnet):

(17) Ie aude / vë̈ghe pluan. (Q2)

"Sento / Vedo piovere."

Con questo tipo di frasi, tuttavia, solo un terzo degli intervistati accetta una proposizione gerundiva, mentre gli altri informatori ricorrono a soluzioni diverse, in particolare a una completiva introdotta da che o coche.

Fin qui, dunque, la situazione è chiara. Ma il gardenese presenta, rispetto alle altre lingue romanze, un'ulteriore innovazione; infatti esiste una possibilità di indicare il soggetto semantico, senza spostare però su di esso l'accento: ${ }^{20}$

(18) Chësc latin che la jënt de nosc luesc audiva rujenan dai mpieghei romans, dai saudeies, dai marciadënc y traficanc ne fova nia chël tlassich [...] (Bels. 0)

"Questo latino che la gente dei nostri posti sentiva parlare dagli impiegati romani, dai soldati, dai mercanti e commercianti non era quello classico [...]."

(19a) Ie vë̈je ciacian l cërf dal iagher. (Q20a)

"Vedo cacciare il cervo dal cacciatore."

(19b) L veïje ciacian dal iagher. (Q20d)

"Lo vedo cacciare dal cacciatore."

In questi casi si assiste all'inserimento di un complemento d'agente per indicare il soggetto semantico, e in questo modo l'evento percepito rimane al centro dell'attenzione. In particolare, in (18) il ricorso a questa struttura può essere dovuto anche al contesto, dal momento che la costruzione percettiva si trova all'interno di una frase relativa, il cui antecedente è rappresentato dall'oggetto del gerundio.

La possibilità di inserire un complemento d'agente nella costruzione percettiva al gerundio costituisce uno dei tratti più originali del gardenese, e rende ancor più evidente l'allontanamento della struttura di questa varietà da quella comune.

${ }^{20}$ Vorrei sottolineare come queste frasi non siano giudicate grammaticali da tutti i parlanti: sono soprattutto quelli più giovani o aperti alle innovazioni ad accettare le frasi in cui il gerundio assume un senso passivo, con o senza complemento d'agente (su questa definizione cf. nota 41). Alcuni di loro, inoltre, accettano anche frasi come Ie l vëjje unian ciacià dal iagher ("Lo vedo venir cacciato dal cacciatore"). I parlanti più conservatori, invece, rifiutano tutte queste strutture. 
Nell'interrogarsi sulle origini di questa innovazione è naturale pensare all'italiano, in cui è possibile utilizzare un complemento d'agente all'interno di un'infinitiva retta da verbo percettivo, oppure nella struttura fattitiva (entrambe le strutture sono di tipo monofrasale):

(20) ... lo sapeva da tempi remoti, forse da quando, bambina, l'aveva vista [la lana] filare dalle vecchie contadine lucane.. $.21^{21}$

(21) Ricevendolo a Villa Savoia, il re comunica al Duce il suo avvenuto licenziamento e all'uscita lo fa arrestare dai carabinieri.22

In effetti, quest'innovazione peculiare del gardenese potrebbe trarre la sua origine dall'influsso della lingua italiana. Ciò sembra provato anche dalla posizione del clitico, che nella frase (19b) corrisponde a quella dei due esempi italiani, mentre in gardenese, partendo dalla frase (19a) ci si aspetterebbe una frase con il complemento oggetto del gerundio cliticizzato, ${ }^{23}$ dove il clitico precede il gerundio: ${ }^{24}$

(22) *Ie veije l ciacian dal iagher.

La differenza tra (20) e (21) però consiste anche nella frequenza d'uso; se infatti l'infinitiva con un complemento d'agente e dipendente da un verbo di percezione non è molto frequente nemmeno in italiano, dove si preferisce una completiva o

${ }^{21}$ Cf. Marghieri 1974, 45 citato da Skytte 1983, 256.

${ }^{22}$ C. Morante 1974, 142 citato da SKYTTe 1976, 371.

${ }^{23}$ H. Siller-Runggaldier (comunicazione personale) ha osservato come questa frase potrebbe essere agrammaticale perché il pronome costituisce il complemento oggetto del verbo di percezione (la stessa interpretazione della struttura è stata proposta anche da SKYTTE 1983, 259-261), e quindi dovrebbe per forza salire prima di questo. Sono d'accordo con quest'interpretazione, ma solo a patto di stabilire che le frasi senza soggetto semantico hanno sempre una struttura monofrasale: di conseguenza, il verbo di percezione e il gerundio formano un blocco unico, e il pronome non può quindi spezzare quest'unità verbale. La stessa osservazione vale peraltro per le frasi che abbiano un $\mathrm{SN}$ al posto del pronome, in gardenese come in italiano: *Ie vë̈je l cërf ciacian dal iagher "*Vedo il cervo cacciare dal cacciatore".

L'agrammaticalità di quest'ultima frase (con struttura bifrasale), cui corrisponde (22), dimostra come anche in gardenese strutture di questo tipo siano possibili solo se monofrasali: in questi casi, infatti, la posizione sintattica di complemento oggetto di tutto il complesso verbale è già occupata da l cërf, e quindi il soggetto semantico, che normalmente costituisce il complemento oggetto del verbo di percezione, dev'essere espresso in un altro modo, ossia come complemento d'agente. La conseguente interpretazione obbligatoria di (18) e (19) come costruzioni monofrasali conferma la mia teoria sull'influsso dell'italiano o della costruzione fattitiva gardenese (es. 23). Per una discussione più approfondita della costruzione monofrasale cf. infra, \4.6.

${ }^{24}$ Sulle possibilità di cliticizzazione e sulle posizioni dei clitici cf. infra, $\int 4.5$. 
pseudorelativa soprattutto nel registro parlato, la struttura fattitiva con complemento d'agente è molto più comune, ed esiste anche in gardenese:

(23) La lëtres fejen scrì da na secretera. (Q64)

"Le lettere si fanno scrivere da una segretaria."

È possibile dunque che qui vi sia un influsso dell'italiano, ma anche che si tratti di un'innovazione basata solo sull'analogia con un tipo di frase gardenese relativamente vicino alla costruzione percettiva. ${ }^{25}$

Per quanto riguarda la funzione grammaticale, infine, il soggetto semantico del gerundio di solito costituisce il complemento oggetto del verbo di percezione; in rari casi, ossia quando quest'ultimo è espresso al passivo, il soggetto del gerundio costituisce contemporaneamente il soggetto del verbo di percezione, come nell'esempio (12), qui ripetuto:

(12) L lere ie unì udù mucian cun I motor. (Q21a)

"Il ladro è stato visto fuggire con la moto."

Non sono ammessi invece soggetti semantici al dativo o preposizionali, perché $i$ tre verbi di percezione che possono essere seguiti dal gerundio sono tutti transitivi, a differenza per esempio di cialé e cialé pro ("guardare").

\subsection{II verbo al gerundio}

Il verbo al gerundio costituisce l'elemento centrale della struttura analizzata in questo lavoro; prima di concentrarci sul gardenese, vorrei ricapitolare brevemente le norme che regolano l'uso della gerundiva in spagnolo e francese. In queste lingue, come abbiamo già potuto vedere, l'uso del gerundio nella struttura percettiva presuppone la presenza di un antecedente con valore di soggetto semantico, e un valore aspettuale preciso: il gerundio, infatti,

erscheint als aspektuell markiert: Es zeigt nämlich Durativität an und kennzeichnet ein Geschehen entsprechend als verlaufend und ohne klare zeitliche Limitierung. Im allgemeinen ist für das Gerundium auch das Merkmal der Gleichzeitigkeit konstitutiv. ${ }^{26}$

\footnotetext{
${ }^{25}$ I casi in cui la costruzione con i verbi di percezione si avvicina alla struttura monofrasale verranno discussi al $₫ 4.6$.

${ }^{26}$ SilLer-RungGaldier 1997, 310.
} 
Se si vuole trasferire quest'osservazione al gardenese, vedremo come essa sia sicuramente valida nella sua seconda parte, dal momento che anche diversi informatori hanno dichiarato di sentire il gerundio principalmente come indicatore di contemporaneità tra i due eventi, quasi come se la gerundiva fosse parafrasabile con una proposizione subordinata introdotta dalla congiunzione mentre. Cercherò di dimostrare invece che la prima parte della citazione può essere riferita al gardenese come una tendenza generale, ma non come una vera e propria norma.

Per analizzare nel dettaglio i verbi che possono essere inseriti in una proposizione gerundiva, bisogna considerare innanzitutto le restrizioni ad alcuni tipi di verbi, che non sono accettati in questa costruzione: si tratta dei verbi modali e degli ausiliari avëi e vester ("avere" e "essere"). ${ }^{27}$ La stessa limitazione si riscontra nelle costruzioni percettive italiane all'infinito, che SKYTTE $(1983,249)$ spiega facendo riferimento alla neutralità di questi verbi rispetto all'Aktionsart ("azione verbale"). Nessun altro tipo di verbo, invece, è soggetto a restrizioni. Per quanto riguarda la valenza verbale, per esempio, sono accettati tutti i tipi di verbi, dagli zerovalenti come piovere (cf. supra) ai trivalenti (ossia i verbi con un complemento oggetto diretto e uno indiretto, come dire):

(24) N di àl audi la usc de Die ti dijan: "Vàten da tlo y va a sté ulache ie te mustreré!" (Bib. pit. VT) "Un giorno ha sentito la voce di Dio dirgli: "Vattene da qui e vai a stare dove io ti mostrerò!" "

Anche i verbi riflessivi possono entrare in una costruzione al gerundio, e in questo caso possono mantenere il pronome riflessivo (25) oppure apparire senza (26):

(25) L mut à udù l giat se lecan, y me à damandà cieche l fajova. (Q32)

"Il bambino ha visto il gatto leccarsi, e mi ha chiesto che cosa faceva."

(26) Chisc ultimi insteies àn audi lamentan truep per via dl bur tëmp. (Un. did. 2)

"Queste ultime estati si è sentito lamentare molto (= si sono sentite molte lamentele) per via del brutto tempo."

Uno dei punti principali nell'analisi della costruzione gerundiva riguarda il suo valore aspettuale. Orientandoci secondo l'uso dello spagnolo e del francese, potremmo aspettarci che anche in gardenese si possa utilizzare una gerundiva solo

\footnotetext{
${ }^{27}$ Si noti come in gardenese non esista nemmeno una forma gerundiva per questi due verbi.
} 
quando essa esprima duratività. In realtà, già un'analisi superficiale delle frasi da noi raccolte mostra come questa varietà ladina ammetta una flessibilità molto più marcata rispetto a quanto accade nelle altre due lingue, al punto da essere compatibile con tutti i tipi di azione verbale. ${ }^{28}$

Alcune frasi tipicamente durativo-continuative, tratte rispettivamente dal corpus e dall'Atlante Linguistico del Ladino Dolomitico (ALD-II), sono per esempio:

(27) "Ma da canche la ie nasciuda ne l'éi mo mëi audida bradlan", ti ova l'oma respundú lessú. (Lëtr.) “ 'Ma da quando è nata non l'ho ancora mai sentita piangere', gli aveva risposto la madre."

(28) L audia sunan la ciampanes. (ALD-II, frase 319, punto 86-Bula)

"Sentiva suonare le campane."

Il verbo usato al gerundio in (27), bradlé ("piangere"), indica un'azione prolungata nel tempo. Si tratta inoltre di un evento non stativo perché il verbo non descrive un'azione ininterrotta: il pianto, infatti, può subire anche molteplici pause. Anche nell'altro esempio manca la cosiddetta "condizione di densità", che BERTINETTO (1991,29-32) indica come prerogativa essenziale per distinguere i verbi stativi da quelli continuativi. Infatti anche l'azione del suonare può essere interrotta e poi ripresa senza pregiudicare l'esito dell'azione stessa.

${ }^{28}$ Cf. Gallmann/Siller-Runggaldier/SitTa 2008, 153: “In dieser Verwendung ist das Gerundium im Ladinischen so verallgemeinert, dass es auch in Verbindung mit punktuellen Verben bzw. punktuellen Ereignissen eingesetzt wird. Das überrascht insofern, als das Gerundium aspektuell im Allgemeinen eine gewisse Dauer impliziert."

L'azione verbale (in tedesco Aktionsart), che va tenuta distinta dall'aspetto: l'aspetto di un verbo dipende principalmente dai diversi tempi verbali, mentre l'azione verbale è legata al significato del verbo e generalmente rimane stabile nelle diverse forme. Quest'ultima è stata studiata principalmente da Zeno VendLER (cf. in particolare ID. 1967), e per l'italiano soprattutto da Pier Marco BerTinetTo. In questo lavoro faremo riferimento principalmente a ID. 1991, in cui i verbi vengono divisi in due gruppi: i verbi durativi e i nondurativi. Un verbo è durativo se riguarda processi che si prolungano nel tempo, mentre nei non-durativi il momento iniziale coincide normalmente con il termine dell'evento. I verbi durativi si suddividono in continuativi (che esprimono una continuità dell'azione, che può però essere interrotta e ripresa varie volte, come guardare, lavorare, piangere) e stativi; questi ultimi possono essere permanenti (che indicano qualità stabili di un soggetto, come assomigliare, essere vecchio, fare il professore) o non-permanenti (indicanti uno stato che può essere interrotto, per esempio vedere, sedere, aver sete). Dei verbi non durativi, invece, fanno parte i verbi puntuali (come stupirsi, spaventarsi, prendere un voto). Un gruppo particolare è formato dai verbi telici, ossia $\mathrm{i}$ verbi che indicano il raggiungimento di una meta. I verbi di questo gruppo possono a loro volta essere durativi, e in questo caso si definiscono risultativi (imparare, lavare una camicia, mangiare una mela), oppure non-durativi (e allora vengono chiamati trasformativi, per esempio partire, arrivare, gettare). Infine, esistono i verbi iterativi, che esprimono un'azione o evento che si ripete nel tempo e che formano un sottogruppo dei continuativi. 
All'interno dei verbi durativi, meritano un cenno particolare le frasi in cui si indica un'azione, che in alcuni casi può essere anche puntuale o risultativa, ma che acquista valore continuativo perché reiterata nel tempo.

(29) 'Maester, nëus n'on udù un paràn ora rie spirc te ti inuem, y nëus ti l'on pruibi, ajache I ne fova nia un di nosc." (Bib. sand. B 1)

"Maestro, noi ne abbiamo visto uno scacciare spiriti cattivi in tuo nome, e noi gliel'abbiamo proibito, perché non era uno dei nostri."

In (29) si osserva un chiaro esempio di verbo puntuale, paré ora ("scacciare"), che acquista un senso continuativo perché il complemento oggetto al plurale indica una quantità indefinita di azioni uguali.

All'interno del gruppo dei verbi durativi, esistono altre due categorie: i verbi stativi (30 e 31) e i verbi risultativi (32). Anche con questi due gruppi il gardenese ammette l'uso della gerundiva, benché gli esempi con verbi stativi siano poco frequenti:

(30) La jënt se fajova marueia a audì rujenan stoms, a udëi moncs varìi, viërces udan inò, lëisc zapan via bel. (Bib. pit. NT)

"La gente si stupiva di sentire parlare i muti, a vedere gli storpi guariti, i ciechi vederci di nuovo, i paralitici andare via [camminando] tranquillamente.”

(31) Per l solit ieles vadeles, ma nouei ei udù la mutans fajan la valëntes. (Q6)

"Di solito sono delle pesti, ma oggi ho visto le bambine fare le brave."

(32) N iëde ie ne vëjje pa mo flurian rojules. (Scioldi y rich.)

"Finora io non vedo fiorire rose."

In (30) è presente un verbo stativo permanente, udan, che si contrappone a zapan via ("andare via camminando"), che invece è un verbo telico risultativo. Più difficile è interpretare rujenan ("parlare”), poiché si potrebbe leggere come la descrizione di una proprietà fisica (corrispondente a "avere di nuovo la facoltà di linguaggio", cf. udan di questo stesso esempio), oppure come una semplice azione continuativa. In (31), invece, viene descritto un evento di tipo diverso, poiché fé l valënt solitamente implica un termine (come è testimoniato anche dalla specificazione ncuei, "oggi”). Si può supporre che si tratti di un verbo stativo non permanente, poiché vi è una condizione di densità che invece manca ai continuativi. L'esempio (32), infine, è una frase proverbiale che contiene un verbo indicante un processo - la fioritura di rose - che può durare diversi giorni. 
I verbi trasformativi condividono con i risultativi il carattere telico, ma sono caratterizzati da una durata più breve, e offrono dunque la descrizione di un repentino mutamento di stato, come risulta dagli esempi:

(33) Lenert jiva for plu suvënzpéa y ngali i udòven belau uni domesdì pian da iló su. (Lëtr.)

"Leonardo la accompagnava sempre più spesso e a un certo punto li si vedeva partire da lì quasi ogni pomeriggio."

(34) Ie é udù sàtana tuman jù dal ciel sciche n tarlui. (Bib. sand. C 1)

"Io ho visto Satana cadere giù dal cielo come un fulmine."

Entrambi gli esempi contengono un verbo non durativo: in (33) si parla dell'azione dell'arrivare da qualche parte, che implica una Aktionsart non durativa e telica. La stessa osservazione vale per l'esempio seguente, in cui la caduta rappresenta un evento improvviso e che implica un cambiamento di stato.

All'interno della categoria dei verbi non durativi esiste un secondo gruppo, che si differenzia dai verbi trasformativi perché non comporta l'idea di uno scopo o di una variazione di stato; si tratta dei verbi puntuali. Vediamo un esempio di questa categoria:

(35) Ma Pilato audian numinan la Galilea, à damandà sce l fova dla Galilea. (Bib. sand. C O)

"Ma Pilato sentendo nominare la Galilea ha chiesto se era della Galilea."

Si tratta di un esempio interessante, perché nel brano originale non c'è nessuna costruzione percettiva ("Ma Pilato, sentendo [ciò], chiese se l'uomo fosse della Galilea"; Lc 23,6). Per rendere più chiara la situazione, il traduttore gardenese ha scelto di specificare quale elemento dell'accusa avesse attratto l'attenzione del magistrato romano facendo ricorso al verbo numiné, che è un verbo di tipo puntuale. In questo modo si indica anche come il passaggio del discorso ripreso da Pilato sia in realtà una parte marginale dell'accusa di sedizione. ${ }^{29}$

Dall'azione verbale va distinta la dimensione più propriamente aspettuale della costruzione gerundiva, che corrisponde a quanto si può osservare per il francese e spagnolo, come si vede da (36):

${ }^{29}$ Alcuni esempi di verbi puntuali sono riportati anche da GaLLmann/Siller-RungGaldier/SitTa 2008, 153. 
(36)

Ie é udù Marco tuman sun fon₹:

“Ho visto Marco cadere per terra." v vs.
Ie é udù Marco tumà sun fon :

vs. Ie é udù Marco che fova tumà sun fonz:

Ie é udù Marco sun fonz:

"Ho visto M. caduto per terra."

"Ho visto M. che era caduto per terra."

"Ho visto M. per terra."

Ciò che differenzia qui la struttura al gerundio dalle altre - participio passato, relativa, sintagma preposizionale - è il grado di compiutezza che essa designa. Con il gerundio si indica infatti un evento colto nel suo svolgimento; le altre strutture qui esemplificate indicano invece tutte un evento di cui si coglie il risultato. Se si vuole dunque sottolineare l'atto stesso della caduta, si sceglierà la costruzione gerundiva (o altri tipi di frase che come la gerundiva permettano di indicare anche la contemporaneità); se si dà più importanza al risultato della caduta si utilizzerà un participio o un'altra struttura dello stesso valore aspettuale.

\subsection{L'ordine degli elementi}

Dopo aver visto le caratteristiche principali dei vari elementi che compongono la costruzione percettiva, prenderemo ora in considerazione la struttura complessiva di questa proposizione per vedere quale posizione ricoprano i suoi vari componenti.

Osserviamo innanzitutto la struttura, più o meno complessa, che si può avere in assenza di cliticizzazioni. La struttura di base è costituita da:

(Soggetto) - verbo di percezione - (complemento oggetto) - gerundio - (altri complementi) ${ }^{30}$

Il complemento oggetto del verbo principale può anche essere posto dopo il gerundio, se non vi sono altri complementi:

(37) Ie vëje Andrea unian / unian Andrea. ${ }^{31}$

"Vedo Andrea arrivare / arrivare Andrea."

${ }^{30}$ Gli elementi posti tra parentesi possono essere assenti. Si noti in particolare che il soggetto può mancare solo con quelle persone per cui non esiste un pronome clitico soggetto, ossia alla prima singolare e alla prima e seconda plurale, cf. SALvi 2000, 13-15 e Anderlan-Obletter 1991, 38.

31 Nel complesso, sembra che ci sia una preferenza per l'anteposizione del gerundio al complemento oggetto del verbo di percezione, quando il verbo nella forma non personale è un intransitivo. Ciò però contribuisce al massimo a fornire una nuance di significato diversa, poiché il centro dell'attenzione è posto sull'elemento posposto. 
(38) Ie vë̈je Andrea cherdan si pere. / *Ie vëje cherdan Andrea si pere.

"Vedo Andrea chiamare suo padre. / Vedo chiamare Andrea suo padre."

Quando il complemento oggetto è più “complesso”, esso viene posto obbligatoriamente dopo il gerundio:

(39) Da iló ora audiven bradlan $n$ pitl mut, che cherdova l'oma. (Bels. O)

"Da lì si sentiva piangere un piccolo bambino, che cercava sua madre."

Se il complemento oggetto invece viene focalizzato o costituisce il tema della frase, può essere sollevato oltre il verbo di percezione:

(40) Ajache ëlla éi ie audí lamentan, se laudan, datrai ence scutan chieta. (L pitl pr.)

"Perché lei ho sentito lamentare [= lamentarsi], lodarsi, talvolta anche stare zitta."

L'ordine "verbo di percezione - gerundio", invece, è fisso, con il verbo principale che deve precedere sempre il gerundio, diversamente da quanto può accadere nell'infinitiva italiana. L'ordine rigido di questi due elementi in gardenese presenta un importante vantaggio, che permette di evitare un'ambiguità che esiste invece in spagnolo e romeno, come si può osservare da questa coppia di esempi:

(41a) Ie é udù Maria unian ora de dlieja.

"Ho visto Maria uscire dalla chiesa (e non: ... uscendo dalla chiesa)."

(41b) Unian ora de dlieja, ei udù Maria = Canche son unì ora de dlieja, ei udù Maria. ${ }^{32}$

"Uscendo dalla chiesa, ho visto Maria = Quando sono uscito dalla chiesa, ho visto Maria."

Il senso della seconda frase è chiaro: il gerundio fa riferimento al soggetto dell'intera frase, e può essere parafrasato da una subordinata introdotta da canche ("quando"). Ma le peculiarità del gardenese riguardano la prima frase: in spagnolo e romeno, infatti, una frase di questo tipo è strutturalmente ambigua, perché il gerundio può riferirsi sia al soggetto sintattico, sia al suo complemento oggetto. In gardenese, invece, l'ordine fisso dei due costituenti prevede - sorprendentemente - che in una frase come (41a) il gerundio possa far riferimento esclusivamente al complemento

${ }^{32}$ La prima frase di (41b) sarebbe scorretta - la grammatica normativa non permette infatti di iniziare una frase con un gerundio, stigmatizzandolo come italianismo (cf. ANDERLAN-OBLETTER 1991, 140); è possibile comunque sentire con una certa frequenza frasi di questo tipo. 
oggetto, come mi è stato confermato da tutti i parlanti che ho interrogato su questo punto (13/15). Nemmeno un'intonazione particolare che tenti di creare una pausa tra l'oggetto e il gerundio rende possibile il secondo tipo di lettura. ${ }^{33}$

Infine, vediamo quale posizione assuma la negazione. Di solito si nega il verbo principale, e in questo caso valgono le regole generali: ne + verbo + nia, oppure $n e+$ verbo ausiliare + nia + participio. ${ }^{34}$ In casi più rari, invece, si può negare il gerundio. Solitamente si tratta dell'espressione di un'azione positiva per mezzo di una litote, come nell'esempio (42), dove l'informazione principale riguarda il fatto che il bambino ha disobbedito:

(42a) Ncuei ei udù l mut dl ujin ne fajan nia l bravo / ne ti ulgan nia a si oma. (Q44)

"Oggi ho visto il bambino del vicino non fare il bravo / non obbedire a sua mamma."

(42b) Ncuei ei udù l mut dl ujin nia ulgan a si mama. (Q44)

"Oggi ho visto il bambino del vicino non obbedire a sua mamma."

In questo caso si ha una discordanza tra gli informatori, dovuta probabilmente anche alla bassa frequenza di una tale costruzione: infatti la maggioranza riprende il modello valido per i verbi nelle forme personali (42a), mentre alcuni preferiscono ricorrere a una negazione semplice, con l'elemento nia posto davanti al verbo negato $(42 \mathrm{~b}) .{ }^{35}$

\subsection{Le possibilità di cliticizzazione}

In questa sezione analizzeremo le possibilità di sostituzione offerte dai pronomi clitici. Tra questi, quelli che rivestono maggiore interesse per la nostra analisi sono

${ }^{33}$ Mentre tutti i parlanti interrogati su questo tipo di frasi hanno negato espressamente che vi sia ambiguità, Bernardi $(1999,187$ ) (in un contesto diverso) cita la frase Ëila à udù l cian jan a spaz. ("Lei ha visto il cane andando a passeggio"), dove il gerundio si riferisce al soggetto del verbo di percezione. È possibile che quando l'azione espressa dal gerundio non è attribuibile al complemento oggetto per motivi semantici (ji a spar, "passeggiare", solitamente è riferito a persone), lo si possa utilizzare anche in questa posizione in dipendenza del soggetto.

${ }^{34}$ Come nel francese standard, in gardenese è obbligatoria la doppia negazione, cf. ANDERLAN-OBLETTER 1988, 128-135.

${ }^{35}$ Questa situazione si può in parte confrontare con il francese, in cui la posizione dell'avverbio di negazione pas dipende dalla forma del verbo: se questo è all'infinito, la negazione precede il verbo, come in (ne) pas voir. Se il verbo invece è espresso in una forma personale o al gerundio, l'avverbio di negazione viene posto dopo il verbo: je (ne) vois pas e (ne) voyant pas. In gardenese l'avverbio nia, preceduto dalla particella ne, segue il verbo flesso, mentre viene posto generalmente davanti al gerundio: nia udan. 
i clitici all'accusativo e al dativo. Se la cliticizzazione è una caratteristica comune e ben radicata in tutte le lingue romanze, vedremo come certe cliticizzazioni, o certe combinazioni di clitici, siano di preferenza evitate dal gardenese nella gerundiva. In generale va comunque ricordato come in questa lingua, a differenza dell'italiano, i pronomi clitici precedano sempre il verbo a cui fanno riferimento. ${ }^{36}$ L'unico pronome clitico usato di frequente nella proposizione gerundiva è il clitico accusativo dipendente dal verbo di percezione, mentre si osserva una certa ritrosia, da parte degli informatori, a cliticizzare i costituenti interni della gerundiva.

La cliticizzazione del complemento oggetto del verbo di percezione è presente in un terzo circa di tutte le frasi del corpus. Gli esempi sono numerosi, e se ne citano due solo a titolo illustrativo:

(43) Ve udon maian cun apetit. (AID-II domanda 489; punto 86-Bula)

"Vi vediamo mangiare con appetito."

(44) Ie sé che Maria ie mò n cuntat cun Piere ajache l'ei ududa ti scrijan. (Q27a; J. D.)

"So che Maria è ancora in contatto con Pietro perché l'ho vista scrivergli."

Per quanto riguarda la posizione, il clitico complemento oggetto si colloca, secondo le regole generali, davanti al verbo di percezione o davanti all'ausiliare se si tratta di un verbo composto.

Un secondo elemento che può essere cliticizzato è il complemento oggetto del gerundio. Si tratta però di un fenomeno assai più raro e poco utilizzato dai parlanti. Nelle mie inchieste l'accettabilità del pronome clitico dipende dal contesto della frase. Quando manca il complemento oggetto del verbo di percezione gran parte degli informatori accetta una frase gerundiva con il clitico anteposto al verbo di percezione:

(45) Ie l vëje ciacian dal iagher. (Q20d)

"Lo vedo cacciare dal cacciatore."

Se invece il complemento oggetto del verbo principale è presente, sia in forma di $\mathrm{SN}$ che di clitico, allora la disponibilità a usare una gerundiva è molto più bassa, come negli esempi seguenti:

\footnotetext{
${ }^{36}$ L'unica eccezione è data dall'imperativo, dove i clitici vengono attaccati al verbo (scrïjëme na cherta da Paris, “scrivetemi una cartolina da Parigi", Anderlan-Obletter 1991, 120).
} 
(46) Ie lé udù la ziplan. (Q10d)

"L'ho visto scolpirla."

(47) ?Ie é udù Marco la ziplan. (Q10b)

"Ho visto Marco scolpirla."

(48) ?? Ie lé udù l fajan. (Q14b; C. B.)

"L’ho visto farlo."

I tre esempi sono posti in ordine di grammaticalità, dal più accettabile a quello ritenuto impossibile da quasi tutti i parlanti. Sul giudizio più negativo riguardante (48) influisce probabilmente la presenza di due clitici che hanno la stessa forma; anche in italiano una frase come ?Lo vedo farlo verrà sostituita normalmente da Glielo vedo fare. La maggiore accettabilità di (46) rispetto a (47) si potrebbe spiegare con la distanza tra i due complementi oggetto nel primo esempio, mentre nel secondo essi si trovano uno di fianco all'altro. Va comunque ricordato come nessuna di queste frasi sia da considerare pienamente accettabile, e il giudizio non migliora se il verbo principale è al passivo, come nel seguente esempio:

(49) 'L cian di uijns ie stat udù les ruban. (Q36b)

"Il cane dei vicini è stato visto rubarle."

Quando nella gerundiva sono invece presenti due clitici, uno oggetto diretto e uno oggetto indiretto, inaspettatamente la grammaticalità delle frasi aumenta, almeno quando il soggetto semantico è un SN:

(50) Ei udù Luca ti la scincan. (Q29b)

"Ho visto Luca regalargliela."

Se invece il soggetto semantico manca (Ti l'ei udù dajan, ${ }^{37}$ "gliel'ho visto dare") o è anch'esso cliticizzato (L ei udì ti l dajan, "l'ho visto darglielo"), le frasi sono giudicate accettabili solo da una minoranza di parlanti.

Infine, vediamo quale grado di accettabilità abbiano i clitici complemento indiretto. In generale, si può notare come essi siano più utilizzati di quelli all'accusativo. La frase (51), infatti, è accettata quasi da tutti: ${ }^{38}$

\footnotetext{
${ }^{37} \mathrm{Si}$ noti come in questo caso gli informatori che hanno tradotto questa frase con un gerundio hanno anteposto i clitici al verbo di percezione, forse per influsso della domanda.

${ }^{38}$ Una frase dello stesso tipo, ma senza soggetto semantico del gerundio (Q38b), è stata invece resa solo dalla metà dei parlanti con una gerundiva.
} 
(51) Inier ei udù Maria ti scrijan. (Q27b)

"Ieri ho visto Maria scrivergli."

I giudizi dei parlanti non cambiano se il soggetto semantico è espresso da un pronome clitico.

Per quanto riguarda la posizione del clitico dativale, esso va inserito sempre immediatamente prima del gerundio; anche quando manca l'oggetto del verbo principale, non è permesso estrarre il clitico dalla sua costruzione (diversamente dal clitico oggetto), come avviene invece in italiano:

\section{(52) *Ti ei udì scrijan. (Q38b) ${ }^{39}$}

"Gli ho visto scrivere."

Riassumendo dunque le possibilità di cliticizzazione, esse vanno dalla piena accettabilità di un pronome clitico in funzione di complemento oggetto del verbo di percezione al rifiuto quasi totale, e in quasi tutti i contesti, per l'espressione dell'oggetto del verbo al gerundio con un clitico. La cliticizzazione del complemento oggetto indiretto invece è accettata da gran parte degli informatori. L'agrammaticalità del clitico oggetto sul gerundio sembra essere dovuta principalmente alla posizione che questo assume nella frase. Infatti, mentre nell'infinitiva italiana i due complementi oggetto rimangono separati a causa della regola che prevede l'enclisi dei modi non finiti (Vedo Maria chiamarlo), in gardenese, dove la proclisi è generalizzata, la cliticizzazione porterebbe all'adiacenza di due complementi oggetto, uno dipendente dal verbo di percezione e l'altro dal gerundio, sentita come elemento di disturbo. Anche quando il complemento oggetto del verbo principale manca o è un clitico (e quindi non si pone più il problema della vicinanza), la lingua gardenese non permette generalmente questo procedimento. È difficile cogliere le cause di questa restrizione; un'ipotesi potrebbe riferirsi al fatto che il posto tra il verbo di percezione e il gerundio sia sentito come riservato al complemento oggetto del verbo principale.

Questa ipotesi sembra confermata da due fattori: l'estrazione del clitico dalla gerundiva e il cumulo di clitici. In precedenza si è potuto notare come l'anteposizione del clitico al verbo di percezione, quando il gerundio è senza soggetto

${ }^{39}$ Due informatori hanno comunque scelto questa frase, ma altrettanti, quando è stata loro proposta, l'hanno rifiutata categoricamente. L'anteposizione del clitico potrebbe dunque essere dovuta all'influsso della domanda (Gli ho visto scrivere). 
semantico, sia una delle poche situazioni considerate del tutto accettabili. In questo caso, infatti, il clitico non occupa il posto dell'oggetto del verbo principale. Una situazione simile si ha con il cumulo di clitici, quando anche il complemento oggetto indiretto viene cliticizzato: in tal caso sembra che si produca uno stacco tra $\mathrm{i}$ due complementi oggetto che rende la frase più accettabile. In definitiva, dunque, ciò che limita a pochi casi la cliticizzazione dell'oggetto del gerundio non sembra essere la cliticizzazione in sé, quanto piuttosto il problema della collocazione del clitico accusativo.

\subsection{Caratteristiche di tipo monofrasale}

Riassumendo le diverse innovazioni del gardenese rispetto alle altre lingue romanze, si può notare che quasi tutte vanno nella stessa direzione, ossia verso la rianalisi della costruzione percettiva come struttura di tipo monofrasale (o fattitivo). ${ }^{40}$ Non si tratta comunque di una modifica che coinvolge la costruzione percettiva tout court, perché la struttura "di base" rimane comunque di tipo bifrasale, come nelle gerundive e infinitive delle altre lingue romanze.

In primo luogo, abbiamo già potuto osservare come in gardenese possa mancare l'oggetto del verbo di percezione, ossia la persona o cosa che è coinvolta nell'evento percepito:

(53) Purdenëde, ma é audì batan, pona m'ei mpensà che sëis tumày ÿ̈is de bujën de aiut. (Laur. deb. 3) "Perdonate, ma ho sentito battere, allora ho pensato che siete caduto e avete bisogno di aiuto."

Se il complemento oggetto manca, il soggetto semantico del gerundio può essere però formulato con un complemento d'agente, come nell'esempio (18) qui riproposto:

\footnotetext{
${ }^{40}$ La costruzione monofrasale viene utilizzata in italiano con i verbi fare e lasciare, e talvolta anche con $\mathrm{i}$ verbi di percezione vedere e sentire. La sua peculiarità principale riguarda i rapporti tra il verbo principale e l'infinito, che vengono a formare un complesso verbale unico, che non può essere separato da alcun costituente e che si comporta come un verbo solo; per questo motivo non possono esserci, per esempio, due complementi oggetto (*Faccio portare Gianni le valigie, ma Faccio parlare Gianni). Nella cosiddetta costruzione bifrasale (o percettiva), invece, i due verbi non formano un'unità (per maggiori dettagli sulle differenze tra le due strutture cf. SKYTTE/SALvi 1991, 497-513).
} 
(18) Chësc latin che la jënt de nosc luesc audiva rujenan dai mpieghei romans, dai saudeies, dai marciadënc y traficanc ne fova nia chël tlassich adurvà dai scritëures y studiëusc [...] (Bels. 0)

"Questo latino che la gente dei nostri posti sentiva parlare dagli impiegati romani, dai soldati, dai mercanti e commercianti non era quello classico adoperato dagli scrittori e studiosi [...]."

In questo caso si può notare come il gerundio assuma un valore semantico di tipo passivo ${ }^{41}$ come è testimoniato appunto dall'impiego di un complemento d'agente al posto del complemento oggetto. Come abbiamo già potuto vedere al $\ 4.2$, in questi ultimi due esempi si pone l'accento sull'evento che è stato percepito, mentre l'agente dell'infinito ha un'importanza minore. Ciò contrasta con quanto accade in altre lingue, dove il gerundio deve sempre far riferimento all'oggetto del verbo di percezione, che quindi deve essere necessariamente presente.

Una seconda caratteristica tipica della costruzione percettiva gardenese riguarda i pronomi clitici. Mentre nelle altre lingue romanze i pronomi clitici dipendenti dal gerundio si collocano in posizione adiacente a quest'ultimo, in gardenese il clitico all'accusativo o riflessivo può essere sollevato in posizione proclitica rispetto al verbo di percezione, come negli esempi:

(54) L (sc. l cërf) vëiji ciacian. (Q20c)

"Lo (sc. il cervo) vedo cacciare."

(55) "Ha, ha, ha!" s'la cuinova Golia a se udë unian de contra cb'lpitl mandl. (Bib. pit. VT)

“" $\mathrm{Ha}$, ha, ha!' se la rideva Golia a vedersi venire incontro quel piccolo ometto."

Questo fenomeno attesta come in gardenese il verbo di percezione possa formare un'unica unità verbale assieme al gerundio, e per questo motivo il pronome si presenta in proclisi al verbo principale. Nelle altre lingue romanze, invece, la presenza obbligatoria del soggetto semantico del gerundio/participe présent (J'ai entendu Paul chantant une chanson "Ho sentito Paolo cantare una canzone", ma *J'ai entendu chantant une chanson, che rende quindi impossibile anche una salita del clitico come nell'esempio [54]) conferma come i due verbi non possano mai formare un'unità. Si noti peraltro come in gardenese la salita del clitico sia possibile solo con il clitico oggetto o riflessivo, mentre con il clitico al dativo i giudizi sono discordanti ("? Ël ti à udù dajan na scincunda, "Lui gli ha visto dare un regalo.").

${ }^{41}$ In questo lavoro ho scelto di utilizzare il termine "tipo passivo" - facendo riferimento a SKYTTE (1976 e 1983) - per indicare le costruzioni che presentano un complemento d'agente, come nell'esempio (18) o in cui manca il complemento oggetto del verbo di percezione, mentre è presente l'oggetto del gerundio (esempi 15a e 16a al § 4.2). Il termine complementare "tipo attivo" indica tutti gli altri casi. 
Infine, un comportamento peculiare del gardenese si osserva anche con i pronomi riflessivi; mentre nella costruzione bifrasale i pronomi riflessivi compaiono regolarmente, si possono trovare anche esempi di questo tipo:

(56) Chisc ultimi insteies àn audi lamentan truep per via dl bur tëmp. (Un. did. 2)

"Queste ultime estati si è sentito lamentare molto per via del brutto tempo."

Qui siamo in presenza di un verbo riflessivo, se lamenté, che però appare privo del pronome. Anche in questo caso la motivazione va individuata nella rianalisi del complesso verbale come un'unità, che in questo modo rende impossibile l'inserzione di un pronome tra i due verbi. Bisogna sottolineare però che in questi casi l'uso di una costruzione monofrasale è solamente opzionale, perché rimane sempre possibile ricorrere a una costruzione bifrasale (in cui i pronomi riflessivi sono obbligatori) come nell'esempio:

(57) Ti ultimi ani àuden suvënz se lamentan che la jënt ne n'ie nia plu nteresseda ala politica. ("La Usc di Ladins" 39, 2010, 12)

"Negli ultimi anni si sente spesso lamentarsi che la gente non è più interessata alla politica."

In gardenese, quindi, le caratteristiche della proposizione gerundiva si discostano parzialmente da quanto si può notare nelle altre lingue romanze che hanno conservato questa costruzione; le innovazioni sembrano andare concordemente verso una strutturazione di tipo monofrasale, ossia una interpretazione dei due verbi non più come unità separate, in cui il gerundio dipende dal verbo di percezione, ma come un unico complesso verbale. ${ }^{42}$ Ciò è confermato dalla salita dei clitici, dalla possibile mancanza di un soggetto semantico del gerundio, dalla possibilità di avere un complemento d'agente e dal comportamento dei verbi riflessivi. Si tratta di alcuni aspetti in prevalenza indipendenti, che nel complesso confermano come sia in atto una riorganizzazione più ampia del sistema dei verbi di percezione, a cui però non tutti i parlanti partecipano allo stesso modo: alcuni di loro hanno mostrato una maggior apertura verso le innovazioni, mentre altri, soprattutto quelli con più alta scolarizzazione e una maggior vicinanza al mondo tedescofono, si sono mostrati più restii. ${ }^{43}$ Ad ogni modo, si può senz'altro osservare una notevole distanza della

\footnotetext{
${ }^{42}$ Bisogna notare come la presenza di un gerundio al posto dell'infinito non impedisca di per sé la rianalisi della struttura come monofrasale; infatti in francese antico era possibile utilizzare il participe (diacronicamente corrispondente al gerundio gardenese) anche con i verbi fattitivi, cf. FoulET 1982, 95-100, che cita tra gli altri l'esempio Et Lancelot le nos a bui fait entendant (Gr. 11, 5-6).

${ }^{43}$ Va segnalata inoltre, seppur non risulti inattesa, la differenza generazionale tra gli anziani, più conservatori nell'uso di questa costruzione, e i giovani che appaiono invece più innovatori.
} 
gerundiva gardenese dalle altre lingue che fanno ricorso al gerundio con i verbi di percezione, principalmente spagnolo e francese.

\section{Conclusioni}

In questo lavoro ho preso in esame un ambito finora poco studiato di una lingua minoritaria e prevalentemente orale come il ladino della Val Gardena, in cui sono state sviluppate diverse strutture alternative per i verbi di percezione. Purtroppo la mancanza di fonti precedenti il 1800 rende molto difficile - se non impossibile - tracciare le linee evolutive che hanno portato alla situazione odierna. Se alcune strutture risalgono al latino volgare e hanno avuto uno sviluppo lineare, altre presentano un'origine più complicata e che può essere ricostruita solo parzialmente.

In ogni caso la sopravvivenza del gerundio rappresenta un elemento arcaico, ereditato direttamente dal latino volgare. Nel confronto con il resto della Romània questo tratto non costituisce però un'esclusiva del gardenese, poiché lo si ritrova anche in lingue come spagnolo, francese e romeno.

La situazione del gardenese assomiglia però solo superficialmente a quella dello spagnolo e del francese. Come fa notare Siller-Runggaldier (1997, 318), in ladino e in romeno il gerundio con i verbi di percezione ha raggiunto "einen höheren Grammatikalisierungsgrad", perché la mancanza dell'infinitiva ha fatto sì che si rinunciasse a determinate caratteristiche del gerundio - la duratività, l'esigenza di un soggetto semantico esplicito - per ottenere una costruzione più duttile, pressoché generalizzata a tutti i contesti. Rispetto alla situazione del latino volgare e di altre lingue romanze la gerundiva ha perso dunque gran parte dei suoi valori semantici e aspettuali, cosicché oggi può essere considerata una costruzione del tutto analoga all'infinitiva italiana o francese.

Ad ogni modo, il sistema dei verbi di percezione non è, per così dire, perfetto, come mostra per esempio la difficoltà di sostituire l'oggetto del verbo al gerundio con un clitico, e determinate caratteristiche, che finora appaiono marginali o accettate solo da una parte dei parlanti (si pensi per esempio alla salita dei clitici dativo e accusativo prima del verbo di percezione). Tutti questi fattori potrebbero essere la spia di una tendenza al cambiamento, e in definitiva di un sistema in bilico. ${ }^{44}$

44 A prima vista, il sistema delle strutture monofrasali sembra essere un settore particolarmente in bilico, perché anche con i verbi a ristrutturazione (come volere o dovere) si assiste a variazioni: mentre la posizione "canonica" prevede che i clitici precedano l'infinito (Daniel uel les cujiné, "Daniel vuole cucinarle"), come in italiano settentrionale, la tendenza odierna sembra privilegiare la salita del clitico al verbo principale, 
Il gardenese odierno quindi ammette il ricorso alla gerundiva con quasi tutti i verbi (esclusi modali e ausiliari), per esprimere sia eventi di tipo attivo, sia quando vi sia un complemento d'agente - passivo. La limitazione principale, invece, è costituita dalle cliticizzazioni, che non sempre sono giudicate grammaticali. Le differenze con l'analoga struttura spagnola e francese sono notevoli: da un lato vi sono due lingue nazionali, codificate già a partire dall'età moderna e che presentano delle rigide limitazioni all'uso della gerundiva. Dall'altro lato, invece, il gardenese, una varietà alpina tramandata esclusivamente come lingua orale fino a pochi decenni fa, in cui i tentativi di normazione sono rimasti finora meno efficaci (anche perché il fine primario di chi si cura della lingua gardenese è (giustamente) la sua difesa dagli influssi esterni e la sua stessa sopravvivenza). In questo modo la gerundiva si è sviluppata in modo più libero, ma con meno sistematicità, e il risultato sono gli atteggiamenti discordanti dei vari parlanti di fronte a una serie di innovazioni.

Infine, bisogna tener conto di un'altra peculiarità del gardenese: mentre la maggior parte delle lingue minoritarie subisce l'influsso e la concorrenza di un'unica lingua di prestigio (è il caso, per esempio, del fassano e livinallese contemporaneo), in gardenese e badiotto le lingue ufficiali e di prestigio sono due, ed entrambe queste lingue hanno agito sul comportamento sintattico dei verbi di percezione. In particolare, mentre l'influsso del tedesco è visibile soprattutto nella completiva formata con la congiunzione coche ("come") e forse nella limitazione della struttura gerundiva a soli tre verbi di percezione, l'italiano sembra aver influito sulla proposizione gerundiva, che è venuta nel tempo a corrispondere sempre più chiaramente all'infinitiva italiana. ${ }^{45}$

La proposizione gerundiva nelle costruzioni percettive costituisce in conclusione un fenomeno assai interessante del gardenese, perché si pone all'incrocio tra arcaicità e innovazione, tra sviluppo autonomo e influssi esterni, tra eredità romanza comune e peculiarità gardenesi. In definitiva, una testimonianza della vitalità di questa lingua minoritaria posta ai margini del grande mondo romanzo.

come nell'italiano meridionale (Daniel les uel cujinê), anche se le grammatiche non prevedono esplicitamente quest'alternativa. Già LARDSCHNEIDER-CiAMPAC (1909, 161-163), però, distingueva tra la "volkstümliche Sprache", in cui il clitico precederebbe sempre all'infinito, e le "Abweichungen" da quest'ultima, che si riscontrerebbero solo nei testi influenzati da modelli stranieri.

45 Personalmente, sono convinto come proprio la posizione di equilibrio tra italiano e tedesco abbia permesso al gardenese - e probabilmente anche al badiotto - di mantenersi così vitale, e che anche in futuro il rischio maggiore per queste varietà sia costituito da uno sbilanciamento eccessivo verso una delle due lingue di prestigio. 


\section{Appendice - Testi del CorpusLad citati in forma abbreviata}

Tra parentesi si indica il nome del documento all'interno della banca dati del CorpusLad.

Bels. 0 / Bels. 1 = Belsarë̈nn, libro di testo per i bambini della quarta e quinta classe elementare (000_Belsarëinn e 001_Belsarëinn).

Bib. pitli NT = La Bibia per i pitli - nuef testamënt, versione del Nuovo Testamento scritta per i bambini (la_bibia_per_i_pitli___nuef_testamënt).

Bib. pitli VT = La Bibia per i pitli - vedl testamënt, versione del Vecchio Testamento scritta per i bambini (la_bibia_per_i_pitli__-_vedl_testamënt).

Bib. sand. B 0 / 1 = La Bibia di Sandis per l'anno liturgico B, con inserzione di commenti e riflessioni in gardenese (000_bibia_di_sandis_ann_B e 001_bibia_di_sandis_ann_B).

Bib. sand. $C 0$ / 1 = La Bibia di Sandis per l'anno liturgico C, con inserzione di commenti e riflessioni in gardenese (000_bibia_di_sandis_ann_C e 001_bibia_di_sandis_ann_C).

Fis. = I fisicheri, traduzione dell'opera teatrale Die Physiker di F. Dürrenmatt (I_fisicheri).

L pitlpr. = L pitlprinz, versione gardenese de Ilpiccolo principe di A. de Saint-Exupéry (L_pitl_prinz). Laur. deb. 3 = Lauron deberiëda 3, fogli di lavoro per la terza classe elementare (lauron_deberieda3).

Lëtr. = Lëtres te $n$ fol, racconto di Rut Bernardi (letres_fol-rut_bernardi).

Lib. rel. $3=$ Liber de religion 3, libro di religione per la terza classe elementare (religion3).

N puech $=$ N puech per sort, raccolta di aneddoti del 1914 (N_puec_per_sort-1914).

Scioldi y rich. $=$ Scioldi $y$ richëza...cuntentëza?, opera teatrale scritta in ladino da Rudi Vinatzer (porta_pa_scioldi_y_richëza).

Sëlva zac. = Sëlva zacan y sën, opera teatrale in tre atti sulla storia di Selva di Tita Demetz (Sëlva_zacan_y_sën).

Un. did. 2 = Raccolta di una decina di unità didattiche per la seconda classe elementare preparata dall'Istitut Pedagogich Ladin di Bolzano (unitàdidatica2).

Le risposte tratte dal questionario sono invece indicate con la lettera "Q" seguita dal numero della domanda, e dall'indicazione delle iniziali dei parlanti che hanno fornito quella risposta, se sono meno di tre. In caso contrario, la risposta è stata fornita da almeno tre informatori. 


\section{Bibliografia}

ALD-II = GoeBL, Hans et al. (eds.): Atlant linguistich dl ladin dolomitich y di dialec vejins, $2^{a}$ pert / Atlante linguistico del ladino dolomitico e dei dialetti limitrofi, $2^{a}$ parte / Sprachatlas des Dolomitenladinischen und angrenzender Gebiete", 2. Teil, Salzburg, in corso di stampa.

Anderlan-Obletter, Amalia: La rujeneda dla oma. Gramatica dl ladin de Gherdëna, Urtijëi 1991.

BEnINCÀ, Paola: L'interferenza sintattica: di un aspetto della sintassi ladina considerato di origine tedesca, in: Cortelazzo, Manlio (ed.), Elementi stranieri nei dialetti italiani. Atti del convegno del C.S.D.I., vol. II, Ospedaletto 1988, 229-239.

Bernardi, Rut: Curs de gherdëina. Trëdesc lezions per mparé la rijeneda de Gherdëina / Dreizehn Lektionen zur Erlernung der grödnerischen Sprache, San Martin de Tor 1999.

Bertinetto, Pier Marco: Il verbo, in: Renzi, Lorenzo/Salvi, Giampaolo (eds.), op. cit., 1991, 13-162.

Casalicchio, Jan/Modena, Francesca, The distribution of a + infinitive after perception verbs in Northern Italy, Università degli Studi di Padova; [manoscritto inedito].

Edelstein, Frieda: Sintaxa geriunz̨iului românesc, Bucureşti 1972.

Egerland, Verner: Frasi subordinate al gerundio, in: SALVI, Giampaolo/RenzI, Lorenzo (eds.): Grammatica dell'italiano antico, Bologna 2010, 903-920.

Foulet, Lucien : Petite syntaxe de l'ancien français, Paris $1982^{13}$.

Galdmann, Peter/Siller-Runggaldier, Heidi/Sitta, Horst, unter Mitarbeit von Mischì, Giovanni/Forni, Marco: Sprachen im Vergleich. Das Verb, Bulsan 2008.

GASSER, Tone: Gramatica ladina por les scores, Balsan 2000.

KUEN, Heinrich: Rätoromanisches bei Oswald von Wolkenstein, in: "Ladinia”, III, 1979, 101-124.

JÄGER, Sonja: Infinitiv oder Gerundium? in: Rovere, Giovanni/Wotjak, Gerd (eds.), Studien zum romanisch-deutschen Sprachvergleich, Tübingen 1993, 303-309.

LAMBeRTZ, Thomas: Gerundiale Konstruktionen im Lateinischen und Französischem, in: DAHMEN, Wolfgang et al. (eds.), Latein und Romanisch. Romanistisches Kolloquium I, Tübingen 1987, 158-196.

Lardschneider-Ciampac, Archangelus: Versuch einer Syntax des Grödnerischen, Wien 1909; [tesi di laurea].

Marghieri, Clotilde: Amati enigmi, Firenze 1974.

Morante, Elsa: La storia, Torino 1974.

PlangG, Guntram A.: Ladinisch um 1630 in Tirol, in: “Zeitschrift für romanische Philologie“, 101, 1985, 90-99.

RENZI, Lorenzo/SALVI, Giampaolo (eds.): Grande grammatica italiana di consultazione, vol. II: I sintagmi verbale, aggettivale, avverbiale. La subordinazione, Bologna 1991.

SALVI, Giampaolo: Il ladino. Schizzo linguistico, in: "Verbum”, 2/1, 2000, 151-169.

SALVI, Giampaolo: La continuità delle strutture morfo-sintattiche latine nelle lingue romanze, Università Eötvös Loránd, Budapest; [manoscritto inedito].

Siller-Runggaldier, Heidi: Perzeptionsverb + Verb im Gerundium', ein im Romanischen funktional einheitliches Konstruktionsmuster?, in: IliEscu, Maria et al. (eds.), Ladinia et Romania. Festschrift für Guntram Plangg zum 65. Geburtstag, Vich - Vigo di Fassa 1997, 309-320. 
ŠKERLJ, Stanko: Syntaxe du participe présent et du gérondif en vieil italien. Avec une introduction sur l'emploi du participe présent et de l'ablatif du gérondif en latin, Paris 1926.

SKYTTE, Gunver: I costrutti infinitivi con i verbi fattitivi e con i verbi di percezione, in: "Studi di grammatica italiana", 5, 1976, 355-399.

SKYTTE, Gunver: La sintassi dell'infinito in italiano moderno, Copenaghen 1983, 2 voll.

SkYtTE, Gunver/SALVI, Giampaolo: Frasi subordinate allinfinito, in: ReNZI, Lorenzo/SALVI, Giampaolo (eds.), op. cit., 1991, 483-569.

TekavČić, Pavao: Grammatica storica dell'italiano, vol. II: Morfosintassi, Bologna 1972.

Vendler, Zeno: Linguistics in Philosophy, Ithaca (New York), 1967.

Sito del CorpusLad: < http://corpuslad.ladintal.it/applications/textanalysis/sitecorpuslad/index. jsp?_VP_V_ID=2838195> (data dell'ultima consultazione: 7.11.2011)

\section{Ressumé}

Chest articul rejona di verbs che dà dant na perzezion sensoriala (p.ej. vedei, audi), y dles strutures sintatiches che an pò adoré empera. Tl gherdeina (y tl badiot, che ne vegn chilò nia analisé) él tipich abiné te chisc caji n gerunde; ence te d'autri idioms romanics, sciche $\mathrm{tl}$ franzeis $\mathrm{y} t \mathrm{spagnol}$, àn chesta poscibelté, ma ilò pòn adoré ence $\mathrm{n}$ infinitif, tl gherdeina no. Perchel à les proposizions gerundives conesciù tl gherdeina na evoluzion particolara, che vegn descrita avisa te chest articul. Les carateristiches plu importantes é che an pò avei na gerundiva zenza si soget semantich, che an pò mete tl gerunde nia demé verbs duratifs, ma belau de vigni sort, $\mathrm{y}$ che te $\mathrm{n}$ valgugn caji se comporta 1 verb de perzezion y 1 gerunde sciche n verb unich (spo dijen che al é na "costruzion monofrasala"). A la fin dles finedes ti someia la gerundiva tl gherdeina a les infinitives dl talian y dl franzeis, ma pervia de si inovazions pel che 1 sistem ne ae nia ciamò abiné na sia balanza, sciche $\mathrm{n}$ valgunes carateristiches che é ries da capì desmostra (sciche les limitazions tla pronominalisazion), y che se muda poester tres ciamò. 